\title{
MINIREVIEW
}

\section{Mechanical, Thermal, and Optical Changes of the Nerve Membrane Associated with Excitation}

\author{
Akira WatanABE \\ National Institute for Physiological Sciences, \\ Okazaki, 444 Japan
}

In this minireview, some of the recent experimental findings on the excitable membranes of nerve fibers obtained by nonelectrophysiological means are discussed. This minireview, however, does not treat the vast literature on the "optical probes of the membrane potential," which is adequately covered by other excellent reviews $[14,22,23,30,52]$.

When the nerve fiber is excited, it transiently changes many of its physical properties. From the biological point of view, the most significant is the change in membrane potential, since the transmission of information along the nerve fiber is mediated by the electric local current. Other physical changes are significant mainly because they contain information on the molecular mechanism of the excitation process. They are usually very small for an easy detection, but progress in the techniques of extracting small signals from noise allowed us to discover and examine some of these signals. In this minireview, several recent findings on mechanical, thermal, and optical signals will be discussed.

\section{MECHANICAL RESPONSES}

In 1950, D. K. HiLl [37] reported that the radius of a cuttlefish giant axon increases when the axon is stimulated repetitively. In 1955, BRYANT and TOBIAS [7] reported that the length of a crab nerve decreases slowly on repetitive stimulation. These findings are now often considered as established, but it would still be worthwhile and interesting to repeat the experiments with modern techniques. Apart from these slow changes, rapid mechanical changes of a nerve, elicited by a brief single stimulus, have also been reported. However, most of the latter kind of changes are now considered inaccurate, because independent trials to repeat the experiments were mostly unsuccessful (see, [14]). Only after introduction of the signal averager, has more reliable data started appearing.

Received for publication June 2, 1986 
In 1977, B. C. HiLl and his associates [35] reported that the diameter of a crayfish giant fiber decreased transiently when the axon was stimulated. Their published record seems clear and convincing, but they noted that the signal sometimes appeared in different shapes. Their initial report was not followed by a full account and the details of their methods are not available. Later experiments by TASAKI and others [60-63] showed that the initial, major change is really an increase, rather than a decrease, of the fiber diameter.

TASAKI and his collaborators [63] first reported on the mechanical response of the nerve in 1980 . Several different methods were employed and the results were all consistent [43]. Independently, TERAKAWA [69, 70] confirmed a part of their results. He further added a new finding that the intracellular pressure of the squid giant axon rises transiently on excitation. It is now established that the rapid mechanical change does take place in the axon. Nevertheless, there are very few workers in this field and may important facets remain to be explored.

\section{A) Methods for detecting mechanical response}

TASAKI and his collaborators [43] used three different methods to detect the mechanical change. The first was the most simple; the beam of light was partly blocked by a piece of the platinum which was placed on the nerve. Stimulation caused a decrease in the intensity of the emerging light, indicating that the nerve swelled transiently. The second method was to utilize a pressure-sensitive piezoelectric sensor. The third was to employ a device (called a "Fotonic sensor") which was made of two bundles of optical fibers. At an end (called the "sensory end"), fiber elements of both bundles were mixed, but at the other end they were separated to form two branches with two ends. From one of the ends the light was introduced, which emerged from the sensory end and reflected back by the sample placed near the sensory end. The reflected light went into the second branch and was detected by a photo-detector placed near the end of the branch. The detected light intensity was a function of the distance between the sample and the sensory end of the fiber-optics (see, [63], Fig. 1).

\section{B) Time course of the mechanical response}

A brief stimulus caused a swelling, which was often followed by a shrinkage. The second, shrinkage phase can become very large under some conditions; its amplitude can become three times as large as the amplitude of swelling (Fig. 1). In unperfused squid axons, the peak of swelling roughly coincided with that of the internally recorded action potential [61]. The peak of shrinkage was also around the peak of the after-hyperpolarization, but the return of the mechanical response to the baseline was often more rapid than the return of the membrane potential. When the axoplasm was removed by intracellular perfusion with a protease-containing solution, the mechanical response became slower than that of the action potential [60]. 

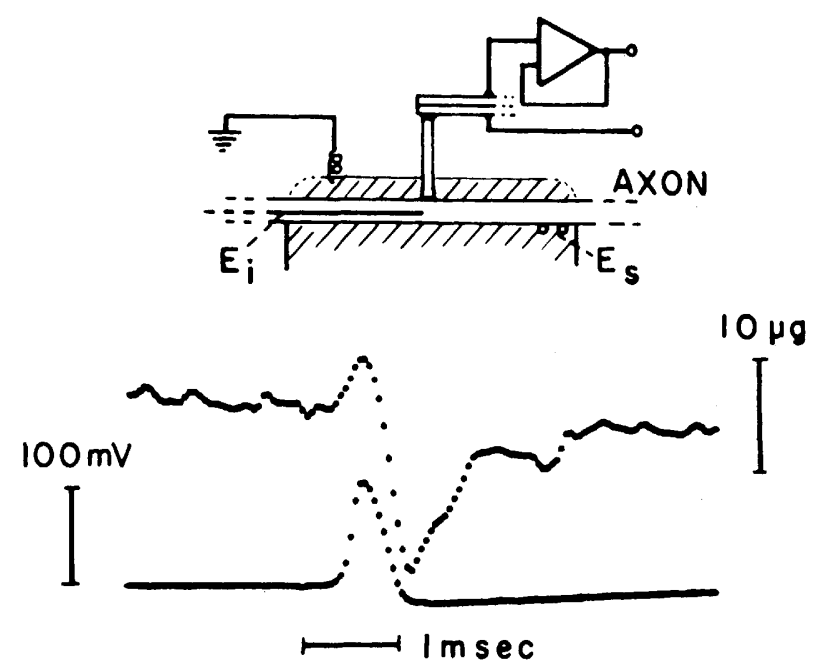

Fig. 1. Top: schematic diagram of the set-up used for demonstrating the degree of timing coincidence between the mechanical and electrical responses of a squid giant axon. A piozo-ceramic bender was held by two pieces of hard rubber and the voltage developed by the bender was measured by a voltage-follower circuit constructed with an operational amplifier. A plastic rod was attached perpendicularly near the free end of the bender. The bender was lowered until the plastic rod was brought into gentle contact with the cleaned axon surface. Es, stimulating electrodes; Ei, intracellular recording electrode. Bottom: upper trace shows the pressure change. Upward deflection shows an increase. Lower trace shows the intracellularly recorded action potential. $23^{\circ} \mathrm{C}$. From TASAKI and IWASA [60].

\section{C) Internal pressure change}

TERAKAWA [70] examined the change in intracellular pressure of the squid giant axon. In his method a glass tubing was connected to an end of the axon and the movement of the water surface at its opening was observed with the "Fotonic sensor." The internal pressure rose when the axon was excited. The rise in the internal pressure was followed by a fall, and the overall time course looked like a mirror image of the mechanical change observed from the external surface. Unfortunately, the time resolution of the records of internal pressure was much worse than that of the externally recorded mechanical change, and the exact correspondence could not be directly established. Nevertheless, the finding supplies an important supplement to the findings obtained by TASAKI and his collaborators [60-63]. It is now apparent that the membrane thickness changes when the nerve is excited. Macroscopically the change looks small (about $1 \mathrm{~nm}$ ), but considering the dimension of the membrane thickness (about $10 \mathrm{~nm}$ ), its importance is clear. It remains to be solved if this thickness change has really nothing to do with the excitation process. 


\section{D) Potential dependence of the mechanical response}

When the crab nerve was depolarized by passing an outward membrane current, swelling took place, and when it was hyperpolarized, shrinkage took place [63]. The response remained even after application of tetrodotoxin (TTX) or procain to suppress excitability. The experiments strongly suggest that the externally recorded mechanical response is determined by the membrane potential change. Mатsumoто et al. [48] recently published several records of swelling of the squid giant axon under the voltage clamp conditions. However, a quantitative relationship between membrane potential and externally recorded mechanical response has still not been worked out.

TERAKAWA [70] performed voltage clamp experiments and determined the relationship between membrane potential and intracellular pressure change. The relationship is quadratic and shows a maximum at about $60 \mathrm{mV}$ of the membrane potential. The curve is very similar to that between membrane potential and birefringence change [16]. This gives rise to the very interesting speculation that the mechanical change and the birefringence change might share a common cause. Whether the parallelism can be maintained under various experimental conditions including the influence of chemicals is worth examining.

The parallelism between membrane potential and mechanical response is not perfect, however. For example, the phase of shrinkage is often much more accentuated than the corresponding after-hyperpolarization, as noted before (see, [70], Fig. 12). A similar discrepancy exists also between membrane potential change and birefringence change [16].

TASAKI and IWASA [62] showed that when tetraethylammonium was injected intracellularly to a squid giant axon so that the action potential formed a plateau, the corresponding mechanical change was not a reproduction of the time course of the membrane potential change. Under similar conditions, however, TERAKAWA [70] showed that the intracellular pressure change followed the time course of the membrane potential. It might be concluded that the mechanical change is a potential-dependent phenomenon in the sense that the membrane potential is an important determining factor of the mechanical change.

\section{E) Drug effects on the mechanical response}

TASAKI et al. [63] showed that the mechanical responses of a crab nerve, elicited by a polarizing current, were not suppressed by TTX or by procain. In the squid giant axon, TERAKAWA [70] found that TTX did not suppress the intra-cellular pressure change either. He however reported that lidocain eliminated the change. The drug concentration was roughly in the range that suppressed excitability. It is probable that suppression of excitability and the mechanical change does not take place accidentally at the same concentration range. They are probably produced from a common cause. One hypothesis which may be worth considering is that lidocain produces a narcotic effect through the change in mechanical properties of 
the axonal membrane. This might lead to a more extreme hypothesis that the permeability change is mediated by the mechanical change. It is true that this sounds very much unlikely, but at present we have little direct evidence to reject it; it can be rejected when we find a chemical with which the mechanical change is suppressed but the excitability is not. In this connection one might recall the existence of a rather conspicuous high pressure effect on the gating current [24]. At any rate, a further search for the effect of drugs on the mechanical response should be performed for the elucidation of the molecular mechanism of excitation.

\section{THERMAL RESPONSE}

The first trial of recording heat production from the nerve was made by Helmholtz in 1848, who found no trace of temperature rise with stimulation of the nerve (see, [29]). A. V. HILL [33] was the first to detect a rise in temperature when the nerve was stimulated repetitively. A rapid temperature change associated with a single stimulus was obtained from a crab nerve about 30 years later by Аввотт $e t$ al. [1]. A surprising discovery of this experiment was that the heat generation was biphasic; after the initial phase of heat production some heat was absorbed by the nerve. The absorption was not confirmed in myelinated fibers, apparently because the quantity of the heat liberated was too small [34]. With the introduction of the signal-averaging technique and with a cautious selection of the experimental material, the accuracy of the measurement was increased considerably. HowARTH $e t$ al. [42] found that the time courses of the corrected temperature change and the monophasically recorded action potential of the garfish olfactory nerve followed almost the same time course. The small discrepancy between the two time courses can be explained if one assumes that the temperature change is proportional to the membrane potential squared and that the membrane potential remains negative even at the peak of the action potential.

The origin of the heat production has been extensively discussed (see, [49]). Among several possibilities the "condenser theory" seems to be the most satisfactory. According to this theory the heat production is a result of discharge of free energy stored in the membrane capacity. The absorption of the heat at the end of the action potential can be explained as a result of recharging the condenser. Probably the dielectrics which form the membrane capacity change their molecular arrangements so that the entropy of the dielectrics changes during the action potential. Only when the entropy change is taken into consideration the chargingdischarging process of the membrane capacity can explain quantitatively the observed amount of heat production.

The detector of the temperature change, used until recently for the study of heat production of the nerve, has almost exclusively been the thermopilegalvanometer combination. At present none of the alternative methods are superior to this device [41]. Nevertheless, it is worthwhile to test other detector-amplifier combinations, since their shortcomings and merits can be recognized only through 
practical applications. TASAKI and IWASA [59] utilized a thermal detector made of polyvinylidene fluoride film in combination with a charge amplifier. An apparent merit of this combination is that it responds to a rapid change in the temperature. The change in temperature of several microdegrees could be recorded from the lobster nerve. It still seems too early to record thermal signals from a single axon preparation. TASAKI and NAKAYE [64] applied their method to squid retina and found a heat production taking place at a very early stage of reaction after the light stimulation.

\section{LIGHT-SCATTERING SIGNALS}

\section{A) Slow changes}

When the nerve is illuminated by a beam of white light and observed at about $90^{\circ}$ to the direction of the incidence the nerve is seen as a bright white thread, showing that the nerve scatters light very strongly. In 1949, D.K. HILL and KEYNES [38] reported that the light scattered by the crab nerve slowly changes its intensity upon repetitive stimulation. The magnitude of the change was in the order of $2 \times 10^{-3}$ of background scattered light. The time course was biphasic; an initial increase in light intensity was followed by a decrease. Later studies showed that the response pattern varied according to the environmental conditions, particularly when the osmotic pressure of the external medium was varied [36]. It also changed according to the grade of the stretch applied on the nerve [6].

\section{B) Rapid changes}

BRYANT and TOBIAS [6] found that a rapid scattering change could be detected in response to a single stimulus, but its details remain unknown. In 1968, CoHEN and KEYNES [17] re-examined the change in scattering, this time with the help of the signal averager. When observed at $90^{\circ}$, a single stimulus produced a transient increase, which was followed by a long-lasting decrease in the intensity of the scattered light from the crab nerve. The later, prolonged phase was sensitive to osmotic pressure and refractive index of the external medium, but the initial transient phase was not much affected by such environmental parameters [18, 19]. The scattering signal can also be recorded from a squid giant axon. The voltage clamp analysis showed that the scattering change has two components: one of them is smaller and dependent on the membrane potential, and the other is larger and slower and dependent on the membrane current [20, 21]. CoHEN [13] expressed disappointment that neither of them was dependent on the membrane permeability, since the optical measurement was originally intended to clarify the structural change of the membrane when its permeability changes upon excitation.

\section{C) Scattering signals from secretory terminals}

The light-scattering signal was small in magnitude and complicated in its origin, and not many studies were performed after the initial exploratory series of 

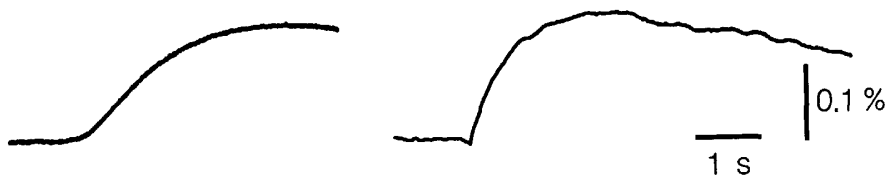

Fig. 2. Changes of light-scattering induced by a single electrical stimulus in neurosecretory nerve terminals of the lobster, Panulirus japonicus (left), and the crayfish, Procambarus clarkii (right). The X-organ sinus gland system was isolated from the eyestalk and the sinus gland region was placed under a bright-field light microscope. The light transmitted through the preparation was monitored by a photodiode. The upward deflection indicates an increase in light intensity detected by the photodiode. The vertical bar represents a fraction of intensity change against background light (Terakawa and Nagano, unpublished).

works. Recently, however, very significant works were published using this optical signal. A large light-scattering signal was discovered from the neurosecretory terminals in the posterior lobe of the mouse pituitary gland [53]. The signal summates and lasts for a considerable period, and the amplitude of the summated signal easily attains the order of $10^{-3}$ of the background light intensity. Pharmacological studies suggest that the signal is most probably associated with the secretory activity of the nerve terminals. The finding would have a large biological significance, since the signal could give us detailed information on the dynamics of neurosecretion.

TERAKAWA and NAGANO [71] recently recorded a large light-scattering signal from the sinus gland on the crustacean eyestalk. The sinus gland is the major crustacean neuroendocrine center analogous with the vertebrate hypothalamicneurohypophyseal system [26]. Electrophysiological studies using intracellular microelectrodes were recently published [25]. The light-scattering change associated with the secretory activity of the sinus gland is probably the largest one so far observed from the neuronal tissue (see, Fig. 2); its size can attain $2 \times 10^{-3}$ of the background light intensity with a single stimulus. The large size facilitated easy analysis of the nature of the signal. The signal was reduced by reducing the extracellular calcium in spite of the small change in size of the action potential. Cadmium also abolishes the light-scattering signal without affecting the action potential greatly.

\section{BIREFRINGENCE SIGNAL}

Light may be represented as a transverse wave of the electric vector (E-vector), but the direction of its vibration from the incandescent light source is randomly and rapidly changing. A polarizer (for example, a sheet of "Polaroid" film) allows only the component of the E-vector which is in parallel with the transmission axis of the polarizer, to pass. If we take two polarizers and cross their transmission axes, no light can go through them. However, if we place a nerve between the crossed polarizers, and turn the nerve so that its longitudinal axis becomes approximately 
$45^{\circ}$ to the transmission axes of the polarizers, the image of the nerve can clearly be observed against the dark background. The phenomenon indicates that the nerve is birefringent. Its longitudinal direction is known to be the "slow axis," that is, the light with its E-vector in parallel with the longitudinal direction of the nerve travels more slowly through the nerve than the light with its E-vector vertical to the longitudinal direction of the nerve. Many cells and tissues exhibit birefringence, because they contain micelles or macromolecules which are arranged in particular directions. The theory of polarized light is briefly described in Davson's textbook [28]. H. S. BENNETT [4] gives a comprehensive account of the theory on polarization microscopy.

\section{A) Resting birefringence}

BEAR et al. [3] examined the birefringence of a squid giant axon with a polarization microscope. They found that the retardation of the fresh axon was positive (i.e., the slow axis is in the longitudinal direction of the axon), and the amount of retardation was about $43^{\circ}$ in phase angle. Schmitr and Schmitr [56] tried to find out if there was any change in the amount of birefringence when the axon was excited, but found no sign of the change. COHEN [14] assumed that if they had used a crab nerve instead of a squid axon, they could have discovered the birefringence signal some 30 years earlier than it was actually discovered by COHEN et al. [19].

\section{B) Transient change}

The birefringence signal is a transient decrease of resting birefringence of the nerve when it is excited. In terms of the ratio between the response and the background light intensity, the birefringence signal is much larger than the scattering signal; the size sometimes is $10^{-3}$ of the background light intensity in the crab nerve, and $10^{-5}$ in the squid giant axon [15]. A detailed analysis of the birefringence signal could therefore be performed using the squid giant fiber [16]. The signal closely follows the time course of the action potential with a small delay which varies with the temperature. With the voltage-clamp analysis the relationship between the membrane potential and the retardation was determined. It could be expressed as a quadratic equation. We have therefore three non-electrophysiological parameters of the axon which are determined by the membrane potential squared: the birefringence change, the heat production and the intracellular pressure change. Clearly, this parameter has an important implication for the axonal membrane; it is proportional to the electrostatic energy of the charge stored across the membrane capacity.

\section{C) Localization of the retardation change}

COHEN et al. [15] recorded the birefringence signals from restricted areas of the enlarged image of a squid giant axon. They could obtain large signals from the area of the axon which was close to the edge, but practically no signals from the central 
part of the axon. The observation suggests that the molecules which produce birefringence signals are arranged radially on the surface of the membrane. SATO et al. [54] re-examined the problem by a modified technique. They removed a part of the axoplasm by suction or, in addition, by using protease, and pressed the axon so that a large part of the axon could be regarded as optically homogenous. A clear birefringence signal could be recorded from the central flattened part. The size of the signal was in the order of $10^{-5}$, which is about the same size as that obtained for an unflattened axon. Clearly the birefringence signal could be recorded from the membrane placed vertically to the direction of the light beam. We therefore have to conclude that the structure producing the birefringence change has an axis of anisotropy longitudinally on the surface of the axon. The structure might be either molecular or supramolecular; the existence of semi-macroscopic structures, such as grooves and foldings of the membrane, cannot be excluded as the cause of anisotropy.

\section{D) Potential-dependence}

The birefringence signal is regarded as a potential-dependent optical signal. The term "potential dependence" means that the shape of the optical signal should be expressed by a single-valued function of the membrane potential. In fact, the birefringence of the squid glant axon may not be regarded as strictly potentialdependent, as is carefully pointed out by COHEN et al. [16]. Especially, the birefringence signal corresponding to the phase of after-hyperpolarization (which will be called the second phase) tends to be more accentuated than the birefringence signal corresponding to the spike of the action potential (which will be called the initial phase). Terakawa and Watanabe observed several times that the second phase became even larger than the initial phase (unpublished work). The tendency differed with the axon and the lack of reproducibility made the search for the cause of this variation difficult.

Another deviation from the strict potential dependence is the existence of a slow birefringence change. In the crab nerve, the birefringence change is very longlasting, as is evident from Fig. 3 of COHEN et al. [19]. With repetitive stimulation the birefringence signal summates, indicating that the signal has a different property from the nerve impulse [80]. A similar, but small, birefringence change is observed in a squid giant axon when stimulated repetitively [78]. At least some part of this longlasting signal originates from the axoplasm, since removal of the axoplasm greatly changes the pattern of this long-lasting part of the birefringence change [79].

\section{E) Colchicin effect}

LANDOWNE et al. [45] found that when the squid giant axon was internally perfused with a perfusate containing a high concentration $(30 \mathrm{mM})$ of colchicine or lumicolchicine, sodium current and the birefringence change associated with the depolarizing pulse were suppressed. The effects of the drugs were reversible to some extent. The authors concluded that at least a portion of the birefringence change is 
associated with the sodium permeability change.

The above experiment is somewhat difficult to interpret because recently colchicine has been found to enhance the effect of hydrostatic pressure of the intracellular perfusate on the membrane excitability [72]. The relationship between membrane potential and retardation response might change with the application of colchicine especially when the hydrostatic pressure applied to the internal perfusate is excessive.

\section{FLUORESCENCE SIGNAL}

\section{A) Intrinsic fluorescence}

Proteins absorb light at about $280 \mathrm{~nm}$ and emit fluorescence with a peak of between 300 and $360 \mathrm{~nm}$. Since the excitable membrane contains channel proteins, the fluorescence from the membrane might change during the excitation of the nerve. However, no reliable report is available on this "intrinsic fluorescence" change (an old report by Ungar and Romano is regarded unreliable; see [14]).

\section{B) Potential probes}

When the nerve is stained by one of the fluorescent dyes, illumination of the nerve with light at the excitation wavelength produces fluorescence from the stained nerve. With the use of proper dyes, the wavelength of this "extrinsic fluorescence" can be brought into the region of the visible light, which is more easily measured than the ultraviolet emission. The extrinsic fluorescence signal was first detected by TASAKI et al. [68]. The usefulness of the signal was greatly increased when Davila et al. [27] found that merocyanine dye gives an extraordinarily large fluorescence signal from the squid giant axon. Since the fluorescence signal follows closely the time course of the membrane potential, the fluorescence signal can be used to monitor the membrane potential change. The dyes employed for this purpose are then called the "potential probes." They report the membrane potential change of cells which are too small to penetrate with the intracellular electrode without inflicting serious injury on them. The principle and the method of application of the potential probe have been extensively reviewed (see, introduction).

\section{C) Orderly structure of axonal membrane}

TASAKI et al. [67] injected a dye 2-p-toluidinylnaphthalene-6-sulfonate (TNS) into a squid giant axon, illuminated it wit a polarized light with its E-vector parallel to the longitudinal direction of the axon, and recorded the fluorescence emitted from the axon by a photodetector placed at $90^{\circ}$ to the direction of the excitation light beam. When a polarizer was inserted in front of the photo-detector, and the axon was stimulated, the fluorescence signal could only be detected if the transmission axis of the polarizer was in parallel with the longitudinal direction of the axon. In other words, the portion of the fluorescence from the axon which constituted the signal appeared to be polarized completely. This phenomenon clearly 
shows that the dye molecules arrange themselves along the longitudinal direction of the axon.

\section{D) Possible fluidity change}

GeorgesCauld and Duclohier [32] examined the fluorescence signals of the pike olfactory nerve stained with pyrene. Illumination of the nerve with $340 \mathrm{~nm}$ light causes fluorescence of the nerve at the visible range. When the nerve is stimulated, emission of fluorescence at $400 \mathrm{~nm}$ transiently increases, whereas emission at $480 \mathrm{~nm}$ transiently decreases. Pyrene is dissolved in the lipid in two forms: a monomer and a dimer called the excimer. The emission spectrum of the monomer has a sharp peak at $390 \mathrm{~nm}$, whereas that of the excimer has a broad peak at $480 \mathrm{~nm}$. The signals obtained with nerve stimulation therefore suggest an increase in monomer concentration and a decrease in dimer concentration. This must be due to a decrease of probability of encounter among dye molecules. On excitation of the membrane, therefore, there must be either a transient increase in local viscosity or alternatively an increase in the volume of the lipid which would dilute the probe molecules. The authors preferred the first interpretation and concluded that the membrane fluidity decreases transiently during excitation.

As the authors stated, the conclusion is still hypothetical. Particularly, it seems unnecessary to abandon their second hypothesis in view of the experimental results obtained by TASAKI et al. [60-63] and TERAKAWA [70], who showed that the membrane thickness increases transiently during excitation.

\section{ABSORPTION SIGNAL}

\section{A) Absorption signal as a potential probe}

Ross et al. [50] found that merocyanine dyes produce not only a large fluorescence signal but also a large absorption signal. The signal again follows the time course of the membrane potential change. Together with the fluorescence signal, the absorption signal is also used as the optical potential probe, and finds a wide area of application in the field of cell physiology.

\section{B) Absorption signals using polarized light}

TASAKI and WARASHINA $[65,66]$ found that the sign of the absorption signal is reversed when the direction of E-vector of the incident light was changed by $90^{\circ}$ under some experimental conditions. For example, a squid giant axon was stained externally with crystal violet, and illuminated with $540 \mathrm{~nm}$ light. With the use of a slit, only the light transmitted through the edge of the giant axon was measured with the photo-detector. When the E-vector of the incident light was in parallel with the longitudinal direction of the nerve, the absorption response was an increase in transmitted light intensity. When the E-vector of the incident light was vertical to the longitudinal direction of the nerve, the response was a decrease in transmitted light intensity. The authors concluded that the dye molecules rotate when the axon 
is excited. Another possible interpretation would be to suppose that the dye molecules attach to the membrane in two different sites or layers. The environment of the dye molecules has to be assumed to differ with the layer of attachment. One advantage of this alternative is the consistency with what has been proposed for the TNS fluorescence signal when the dye is injected intracellularly and excited with polarized light $[66,67]$.

\section{OPTICAL ACTIVITY SIGNALS}

A substance is said to be optically active if it rotates the plane of linearly polarized light passing through it. An optically active substance contains molecules or supramolecular structures which lack a certain aspect of symmetry; they cannot be superimposed to their own mirror images [5]. Nearly all biological molecules are optically active not only because of the existence of asymmetric carbon atoms but also because of foldings (such as helices) of primary chains ([44], p. 17). Change in optical activity is therefore a very sensitive indicator of the change in conformation of the macromolecules ([31], p. 573). Since it is widely held that the excitation is a result of conformational change of the membrane channel molecules (see, e.g., [39], p. 331), it seems worth examining if the optical activity of the nerve changes during excitation.

The general concept and usefulness of the optical activity measurement can best be understood from descriptions in textbooks of biophysical chemistry (e.g., [9], [31]). Lowry's classical book [46] supplies a sound basis for the theory and application of the phenomenon. The molecular origin of the optical activity is a subject of quantum chemistry (e.g., [8], [12]). Many applications to both organic and biological chemistry are compiled in SNATZKE's book [57]. Optical activity of proteins is discussed in JiRgensons [44], and CANTOR and TiMASHEFF [10], among others. Optical activity measurement of membrane fragments or vesicles is reviewed by Wallach and Winzler [75] and Holzwarth [40]. A brief review by Maestre [47] covers the recent developments of the theory, methods, and instrumentation.

WATANABE [77] constructed an apparatus to record rapid optical activity changes. Calibration with sucrose solution indicated that the apparatus could detect the change in optical rotation of about $10^{-3}$ degrees with a time constant of $0.4 \mathrm{~ms}$ (here the time constant represents the time to attain $63 \%$ of the final value from the baseline when a step function is applied to the input). When a lobster nerve was employed as the sample, stimulation produced a signal, an example of which is shown in Fig. 3. The signal had a rapid time course, with a duration comparable to the rising phase of the birefringence signal. Often a slow signal was superposed, but this came from the birefringence change, because it changed its size and polarity when rotating the nerve by about $1-2$ degrees. The optical activity signal was insensitive to the rotation. The experiment shows that a rapid and transient conformational change takes place in the membrane macromolecules at an early stage of excitation. 


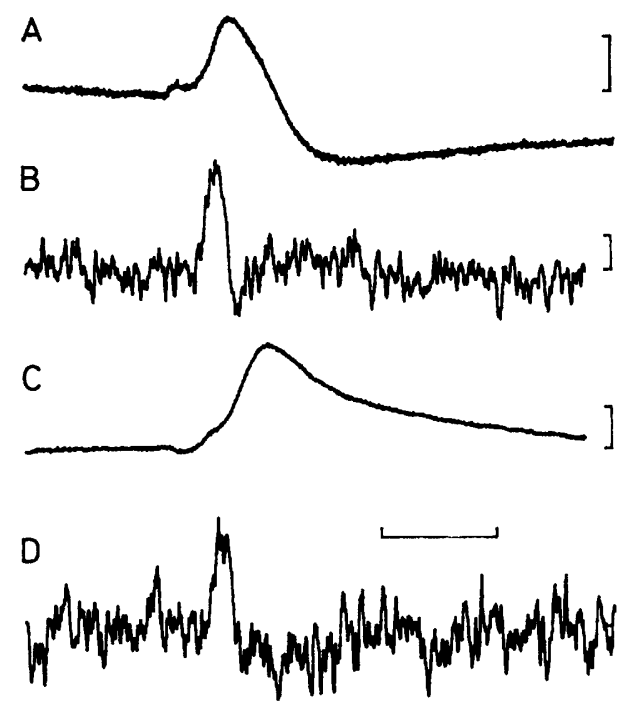

Fig. 3. Optical signals recorded from a nerve dissected from the walking leg of a lobster. A, scattering signal taken at $0^{\circ}$. Upward deflection indicates decrease in light intensity. Vertical bar represents $5 \times 10^{-6}$ of background light intensity. B, optical activity signal. Upward deflection indicates increase in levorotation. Vertical bar represents about $10^{-4}$ degrees. C, birefringence signal. Upward deflection indicates decrease in resting birefringence. Vertical bar represents $10^{-4}$ of background light intensity. D, optical activity signal, recorded again. External medium was natural sea water containing $33 \mu \mathrm{m}$ Merbromin. Time, $10 \mathrm{~ms}$. Wavelength, $570 \mathrm{~nm} .18^{\circ} \mathrm{C}$. From WatanaBE [77].

If an increase in dextrorotation is called a positive response, the optical activity signal from the lobster nerve was either positive or negative or biphasic (mostly positive-negative sequence). One possible explanation for this diversity of signal direction is that polarity of optical rotation depends on the propagation direction of incident light with respect to the orientation of the axes of target molecules (see, [12], p. 295; [73]). Probably, the direction of the optical activity signal is determined by such subtle experimental conditions as the inclination of the nerve with respect to the direction of incident light, or the tension applied to the nerve, which would change the direction of some of the channel macromolecules.

Similar optical activity signals could also be recorded from squid giant axons, but the size of the signal was about one-tenth of that of the lobster nerve [76]. It is necessary to further refine the apparatus to increase the signal-to-noise ratio for its detailed analysis.

\section{RAMAN SPECTROSCOPY}

When a sample is illuminated by an intense source of monochromatic light, the 
scattered light can be observed at right angles to the direction of illumination. If the scattered light is analyzed with a spectrograph, Rayleigh scattering produces a strong spectral line at the wavenelgth of the incident light. At both sides of the Rayleigh scattering, very faint lines can also be observed. These constitute the spectrum of Raman scattering. Raman scattering originates from the interatomic vibrations and rotations of molecules, as infrared absorption does. Since their mechanisms of appearance are different, their spectra show significant differences, but for larger biological molecules both methods give almost identical spectra. The infrared absorption method is, however, not easily applicable to a living preparation, because most of the important absorption bands are masked by strong absorption by water. The Raman spectrum does not have this shortcoming. On the other hand, the bands of the Raman spectrum are very weak in intensity. If the incident light is not purely monochromatic, they are easily covered by the band of the Rayleigh scattering. The difficulty has been much relieved through the introduction of lasers as the Raman excitation source. Application of the Raman scattering to biological systems therefore started developing only recently.

Apart from descriptions in the textbooks of biophysical chemistry (e.g., [9], [31]), more comprehensive accounts are available in monographs and reviews on application of Raman spectra on the biological molecules [11, 51, 74].

Raman spectra of proteins are sensitive to conformation of the protein molecules. Therefore we can expect, as in the case of the optical activity, that some of the Raman bands from the nerve might change when the nerve is excited. If one could detect such a change, the "Raman signal" thus obtained would be more informative than the optical activity signal, since the responsible interatomic vibrations could be identified from the wave number.

A result of such a trial was reported by SzalonTaI et al. [58]. Frog sciatic nerve was dissected and illuminated with a laser beam at $514.5 \mathrm{~nm}$. The Raman spectra were recorded with and without stimulation applied to the nerve at a frequency of $200 \mathrm{~Hz}$. Among changes observed in Raman bands, the ratio $I_{1520} / I_{1157}$ was decreased by some $10 \%$ upon stimulation $\left(I_{1520}\right.$ and $I_{1157}$ denote the band intensities at 1,520 and $1,157 \mathrm{~cm}^{-1}$, respectively). However, SAvole et al. [55] recently repeated the experiment using pike olfactory nerve, and failed to confirm the findings.

The discrepancy in the results might be ascribed to the difference in the experimental material. Nevertheless, it seems necessary to repeat the experiment of Szalontal et al. [58] before the existence of the Raman signal at this order of magnitude is considered as established. One would feel that a $10 \%$ change is too large for an optical signal from the nerve. At any rate, the change should be displayed on the time domain together with the action potential.

Aizawa et al. [2] examined the Raman spectrum of a crayfish abdominal nerve cord. Some of the Raman bands seemed to change when the external medium was changed from a physiological saline to a potassium-rich solution. Among many changes described by the authors, the appearance of a $1088 \mathrm{~cm}^{-1}$ band on 
potassium depolarization seems to be clearest. Unfortunately, experimental data are still plagued by noise and the curved baseline. Further improvement of the apparatus might produce cleaner signals.

\section{CONCLUSION}

Recent advancement in the techniques of detecting non-electrical signals from the nerve fiber is impressive. The recording of absorption and fluorescence signals has now become much easier and found a large field of application. The recording of the thermal signal has been refined to the extent that no further improvement can easily be foreseen. The existence of the mechanical response has been established beyond doubt. It must be stressed, however, that the original purpose of the pioneering workers has not yet been achieved. They started the optical experiments to obtain information on the activity of membrane macromolecules.

At present, signals which are theoretically more intimately connected with the molecular conformational change invariably suffer from a low signal-to-noise ratio. As ConTI [23] argued, signals originating from the channel molecules might be small because of the low density of the channel in the membrane and because of the subtle nature of the molecular conformational change of the channel during excitation. Nevertheless, the present difficulty comes from technical reasons rather than theoretical inaccessibility. For example, a slight improvement of intensity and stability of light sources would be enough to solve many of the obstructing difficulties. With the rapid development of optoelectronics, the molecular conformational change in the axonal membrane may become analyzable independently of the electrophysiological means in the near future.

\section{SUMMARY}

Nerve fibers produce several non-electrical signals on excitation. Recent findings on such non-electrical signals are discussed, in the hope that some of them might give us clues to molecular events underlying the excitation process. At present this expectation is not fulfilled, but progress is being made which allows one to look optimistically at future developments.

Key words: nerve, excitation, mechanical signals, thermal signals, optical signals.

The author thanks Dr. S. Terakawa for his reading the manuscript and making suggestions, and Drs. S. Terakawa and Misako Nagano for permission to use some of their unpublished results.

\section{REFERENCES}

1. Aвbott, B. C., Hill, A. V., and Howarth, J. V. (1958) The positive and negative heat production associated with a nerve impulse. Proc. R. Soc. Lond. B, 148: 149-187. 
2. Aizawa, K., Sakai, S., Imagawa, M., Saito, H., and O'hata, S. (1983) Analysis of phospholipid on the nerve membrane by the micro-Raman spectrophotometer. Bull. Tokyo Med. Coll., 41: 783-788 (in Japanese. Abstract and figure legends in English).

3. Bear, R. S., Schmitt, F. O., and Young, J. Z. (1937) The ultrastructure of nerve axoplasm. Proc. R. Soc. Lond. B, 123: 505-519.

4. BenNeTt, H. S. (1950) The microscopical investigation of biological materials with polarized light. In: McClung's Handbook of Microscopical Technique, 3rd ed., ed. by JoNes, R. M., Hafner, New York, pp. 591-677.

5. Bentley, R. (1969) Molecular Asymmetry in Biology, Vol. 1, Academic Press, New York, $566 \mathrm{pp}$.

6. Bryant, S. H. and Tobias, J. M. (1952) Changes in light scattering accompanying activity in nerve. J. Cell. Comp. Physiol., 40: 199-219.

7. BRyant, S. H. and Tobias, J. M. (1955) Optical and mechanical concomitants of activity in Carcinus nerve. J. Cell. Comp. Physiol., 46: 71-95.

8. Caldwell, D. J. and Eyring, H. (1971) The Theory of Optical Activity, WileyInterscience, New York, $244 \mathrm{pp}$.

9. Cantor, C. R. and Schimmel, P. R. (1980) Biophysical Chemistry, Freeman, San Francisco, $1371 \mathrm{pp}$.

10. Cantor, C. R. and TimashefF, S. N. (1982) Optical spectroscopy of proteins. In: The Proteins, 3rd ed., ed. by Neurath, H. and Hill, R. L., Academic Press, New York, Vol. 5, pp. 145-306.

11. Carey, P. R. (1982) Biochemical Applications of Raman and Resonance Raman Spectroscopies, Academic Press, New York, $262 \mathrm{pp}$.

12. Charney, E. (1979) The Molecular Basis of Optical Activity, Wiley, New York, 364 pp.

13. COHEN, L. B. (1970) Light scattering changes during axon activity. In: Permeability and Function of Biological Membranes, ed. by Bolis, L., KatchalsKy, A., KeYNes, R. D., Loewenstein, W. R., and PethicA, B. A., North-Holland, Amsterdam, pp. 318325 .

14. COHEN, L. B. (1973) Changes in neuron structure during action potential propagation and synaptic transmission. Physiol. Rev., 53: 373-418.

15. Cohen, L. B., Hille, B., and Keynes, R. D. (1970) Changes in axon birefringence during the action potential. J. Physiol. (Lond.), 211: 495-515.

16. Cohen, L. B., Hille, B., Keynes, R. D., Landowne, D., and Rojas, E. (1971) Analysis of the potential-dependent changes in optical retardation in the squid giant axon. J. Physiol. (Lond.), 218: 205-237.

17. Cohen, L. B. and Keynes, R. D. (1968) Evidence for structural changes during the action potential in nerves from the walking legs of Maia squinado. J. Physiol. (Lond.), 194: $85 \mathrm{P}-86 \mathrm{P}$.

18. Cohen, L. B. and KeYnes, R. D. (1971) Changes in light scattering associated with the action potential in crab nerves. J. Physiol. (Lond.), 212: 259-275.

19. Cohen, L. B., Keynes, R. D., and Hille, B. (1968) Light scattering and birefringence changes during nerve activity. Nature, 218: 438-441.

20. Cohen, L. B., Keynes, R. D., and Landowne, L. (1972) Changes in light scattering that accompany the action potential in squid giant axons: Potential-dependent components. J. Physiol. (Lond.), 224: 701-725.

21. Cohen, L. B., Keynes, R. D., and Landowne, D. (1972) Changes in axon light scattering that accompany the action potential: Current-dependent components. $J$. Physiol. (Lond.), 224: 727-752. 
22. Cohen, L. B., Landowne, B., Loew, L. M., and Salzberg, B. M. (1984) Optical signals: Changes in membrane structure, recording of membrane potential, and measurement of calcium. Curr. Top. Membr. Trans., 22: 423-443.

23. Conti, F. (1975) Fluorescent probes in nerve membranes. Annu. Rev. Biophys. Bioeng., 4: 287-310.

24. Conti, F., Inoue, I., Kukita, F., and Stuhmer, W. (1984) Pressure dependence of sodium gating currents in the squid giant axon. Eur. Biophys. J., 11: 137-147.

25. COOKE, I. M. (1985) Electrophysiological characterization of peptidergic neurosecretory terminals. J. Exp. Biol., 118: 1-35.

26. Cooke, I. M. and Sullivan, R. E. (1982) Hormones and neurosecretion. In: The Biology of Crustacea, ed. by Atwood, H. and Sandeman, D., Academic Press, New York, Vol. 3, pp. 205-290.

27. Davila, H. V., Salzberg, B. M., Cohen, L. B., and Waggoner, A. S. (1973) A large change in axon fluorescence that provides a promising method for measuring membrane potential. Nature (New Biol.), 241: 159-160.

28. Davson, H. (1970) A Textbook of General Physiology, 4th ed., Churchill, London, $1694 \mathrm{pp}$.

29. Feng, T. P. (1936) The heat production of nerve. Ergeb. Physiol., 38: 73-132.

30. Freedman, J. C. and Laris, P. C. (1981) Electrophysiology of cells and organelles: Studies with optical potentiometric indicators. Int. Rev. Cytol. (Suppl.), 12: 177-246.

31. Freifelder, D. (1982) Physical Biochemistry, Freeman, San Francisco, 761 pp.

32. Georgescauld, D. and Duclohier, H. (1978) Transient fluorescence signals from pyrene labeled pike nerves during action potential. Possible implications for membrane fluidity changes. Biochem. Biophys. Res. Commun., 85: 1186-1191.

33. HILl, A. V. (1926) The heat production of nerve. J. Pharmacol. Exp. Ther., 29, 161165.

34. Hill, A. V. and Howarth, J. V. (1958) The initial heat production of stimulated nerve. Proc. R. Soc. Lond., B, 149: 167-175.

35. Hill, B. C., Schubert, E. D., Nokes, M. A., and Michelson, R. P. (1977) Laser interferometer measurement of changes in crayfish axon diameter concurrent with action potential. Science, 196: 426-428.

36. HILL, D. K. (1950) The effect of stimulation on the opacity of a crustacean nerve trunk and its relation to fibre diameter. J. Physiol. (Lond.), 111: 283-303.

37. HILL, D. K. (1950) The volume change resulting from stimulation of a giant nerve fibre. J. Physiol.(Lond.), 111: 304-327.

38. Hill, D. K. and Keynes, R. D. (1949) Opacity changes in stimulated nerve. $J$. Physiol. (Lond.), 108: 278-281.

39. Hille, B. (1984) Ionic Channels of Excitable Membranes, Sinauer, Sunderland, 426 pp.

40. Holzwarth, G. (1972) Ultraviolet spectroscopy of biological membranes. In: Membrane Molecular Biology, ed. by Fox, C. F. and KeITH, A. D., Sinauer, Stamford, pp. 228-286.

41. Howarth, J. V. (1970) The technique of thermal measurements in excitable tissues. Q. Rev. Biophys., 3: 429-458.

42. Howarth, J. V., Ritchie, J. M., and StagG, D. (1979) The initial heat production in garfish olfactory nerve fibres. Proc. R. Soc. Lond., B, 205: 347-367.

43. IWASA, K., TASAKI, I., and GibBONS, R. C. (1980) Swelling of nerve fibers associated with action potentials. Science, 210: 338-339.

44. Jirgensons, B. (1973) Optical Activity of Proteins and Other Macromolecules, 2nd 
ed., Chapman \& Hall, London, 199 pp.

45. Landowne, D., Larsen, J. B., and Taylor, K. T. (1983) Colchicine alters the nerve birefringence response. Science, 220: 953-954.

46. LOWRY, T. M. (1935) Optical Rotatory Power, Longmans, London (reprinted by Dover, New York, 1964), 483 pp.

47. Maestre, M. F. (1984) Circular dichroism. In: Optical Techniques in Biological Research, ed. by Rousseau, D. L., Academic Press, New York, pp. 291-341.

48. Matsumoto, G., IchiKawa, M., Urayama, M., Tsukita, S., Tsukita, S., and KobayashI, T. (1986) Subaxolemmal cytoskeleton in squid giant axons and its possible physiological function. Biophysics (Kyoto), 26: 55-66 (in Japanese. Abstract in English).

49. RitchiE, J. M. (1973) Energetic aspects of nerve conduction: The relationships between heat production, electrical activity and metabolism. Prog. Biophys. Mol. Biol., 26: $149-187$.

50. Ross, W. N., Salzberg, B. M., Cohen, L. B., and Davilla, H. V. (1974) A large change in dye absorption during the action potential. Biophys. J., 14: 983-986.

51. Rousseau, D. L. and Ondrias, M. R. (1984) Raman scattering. In: Optical Techniques in Biological Research, ed. by Rousseau, D. L., Academic Press, New York, pp. 65-132.

52. SAlzberG, B. M. (1983) Optical recording of electrical activity in neurons using molecular probes. In: Current Methods in Cellular Neurobiology, 3, Electrophysiological and Optical Recording Techniques, ed. by BARKER, J. L. and MCKelvy, J. F., Wiley, New York, pp. 139-187.

53. Salzberg, B. M., Obaid, A. L., and Gainer, H. (1985) Large and rapid changes in light scattering accompany secretion by nerve terminals in the mammalian neurohypophysis. J. Gen. Physiol., 86: 395-411.

54. Sato, H., Tasaki, I., Carbone, E., and Hallett, M. (1973) Changes in axon birefringence associated with excitation: Implications for the structure of the axon membrane. J. Mechanochem. Cell Motility, 2: 209-217.

55. Savoie, R., Pigeon-Gosselin, M., Pezolet, M., and Georgescauld, D. (1986) Effect of action potential on the Raman spectrum of the pike olfactory nerve. Biochim. Biophys: Acta, 854: 329-333.

56. Schmitt, F. O. and Schmit, O. H. (1940) Partial excitation and variable conduction in the squid axon. J. Physiol. (Lond.), 98: 26-46.

57. SnatzKe, G. (1967) Optical Rotatory Dispersion and Circular Dichroism in Organic Chemistry, Heyden, London, 416 pp.

58. Szalontai, B., Bagyinka, Cs., and Horvath, L. I. (1977) Changes in the Raman spectrum of frog sciatic nerve during action potential propagation. Biochem. Biophys. Res. Commun., 76: 660-665.

59. TASAKI, I. and IWASA, K. (1981) Temperature changes associated with nerve excitation: Detection by using polyvinylidene fluoride film. Biochem. Biophys. Res. Commun., 101: 172-176.

60. TASAKI, I. and IWASA, K. (1982) Rapid pressure changes and surface displacements in the squid giant axon associated with production of action potentials. Jpn. J. Physiol., 32: 69-81.

61. TASAKI, I. and IWASA, K. (1982) Further studies of rapid mechanical changes in squid giant axon associated with action potential production. Jpn. J. Physiol., 32: 505-518.

62. TASAKI, I. and IWASA, K. (1983) Axolemma-ectoplasm complex and mechanical responses of axon membrane. In: Structure and Function in Excitable Cells, ed. by 
Chang, D. C., Tasaki, I., Adelman, W. J., Jr., and Leuchtag, H. R., Plenum, New York, pp. 307-319.

63. TASAKI, I., IWASA, K., and GibBons, R. C. (1980) Mechanical changes in crab nerve fibers during action potentials. Jpn. J. Physiol., 30: 897-905.

64. TASAKI, I. and NAKAYE, T. (1985) Heat generated by the dark-adapted squid retina in response to light pulses. Science, 227: 654-655.

65. TASAKI, I. and WARASHINA, A. (1976) Fast and slow rotation of dye molecules in squid axon membrane during excitation. Proc. Jpn. Acad. Sci., 52: 37-40.

66. TASAKI, I. and WARASHINA, A. (1976) Dye-membrane interaction and its changes during nerve excitation. Photochem. Photobiol., 24: 191-207.

67. Tasaki, I., Watanabe, A., and Hallett, M. (1971) Properties of squid axon membrane as revealed by a hydrophobic probe, 2-p-toluidinylnaphthalene-6-sulfonate. Proc. Natl. Acad. Sci. U.S.A., 68: 938-941.

68. Tasaki, I., Watanabe, A., Sandlin, R., and Carnay, L. (1968) Changes in fluorescence, turbidity, and birefringence associated with nerve excitation. Proc. Natl. Acad. Sci. U.S.A., 61: 883-888.

69. TERAKAWA, S. (1983) Changes in intracellular pressure in squid giant axons associated with production of action potentials. Biochem. Biophys. Res. Commun., 114: 1006-1010.

70. Terakawa, S. (1985) Potential-dependent variations of the intracellular pressure in the intracellularly perfused squid giant axon. J. Physiol. (Lond.), 369: 229-248.

71. Terakawa, S. and Nagano, M. (1986) Scattering change in the neurosecretory terminal. J. Physiol. Soc. Jpn., 48: 190.

72. Terakawa, S. and Nakayama, T. (1985) Are axoplasmic microtubules necessary for membrane excitation? J. Membr. Biol., 85: 65-77.

73. Tinoco, I., Jr. (1959) The optical rotation of oriented helices. I. Electrical orientation of poly- $\alpha$-benzyl-L-glutamate in ethylene dichloride. J. Chem. Phys., 81: 1540-1544.

74. Tu, A. T: (1982) Raman Spectroscopy in Biology: Principles and Applications, Wiley, New York, 488 pp.

75. Wallach, F. H. and Winzler, R. J. (1974) Evolving Strategies and Tactics in Membrane Research, Springer, Berlin, pp., 231-261.

76. Watanabe, A. (1985) The optical activity signal recorded from a squid giant fiber. J. Physiol. Soc. Jpn., 47: 402.

77. Watanabe, A. (1986) Optical activity signals recorded from nerve fibers using a photoelastic modulator. Biomed. Res. (Suppl.), 7: 21-25.

78. Watanabe, A. and Terakawa, S. (1976) A long-lasting birefringence change recorded from a tetanically stimulated squid giant axon. J. Neurobiol., 7: 271-286.

79. Watanabe, A. and Terakawa, S. (1976) Alteration of birefringence signals from squid giant axons by intracellular perfusion with protease solution. Biochim. Biophys. Acta, 436: 833-842.

80. Watanabe, A., Terakawa, S., and Nagano, M. (1973) Axoplasmic origin of the birefringence change associated with excitation of a crab nerve. Proc. Jpn. Acad., 49: $470-475$. 